\title{
Calendar Effects in AAPL Value-at-Risk
}

\author{
Hong-Kun Zhang, Zijing Zhang \\ Department of Mathematics and Statistics, University of Massachusetts Amherst, Amherst MA 01003, USA.
}

Received: March 08, 2016 / Accepted: April 06, 2016 / Published: June 25, 2016.

\begin{abstract}
This study investigates calendar anomalies: day-of-the-week effect and seasonal effect in the Value-at-Risk (VaR) analysis of stock returns for AAPL during the period of 1995 through 2015. The statistical properties are examined and a comprehensive set of diagnostic checks are made on the two decades of AAPL daily stock returns. Combing the Extreme Value Approach together with a statistical analysis, it is learnt that the lowest VaR occurs on Fridays and Mondays typically. Moreover, high Q4 and Q3 VaR are observed during the test period. These results are valuable for anyone who needs evaluation and forecasts of the risk situation in AAPL. Moreover, this methodology, which is applicable to any other stocks or portfolios, is more realistic and comprehensive than the standard normal distribution based VaR model that is commonly used.
\end{abstract}

Keywords: Risk Measures, Value-at-Risk, Extreme value theory, Generalized Pareto Distribution, Day-of-the-week effect, Seasonal effect

\section{Introduction}

Into the maelstrom of digital revolution came a greatly innovative digital company: Apple Inc.. The company designs, manufactures, and markets mobil communication, media devices, personal computers, and portable digital music players, and sells a variety of related software, services, accessories, networking solutions, and third-party digital content and applications. America's favorite pastime used to be baseball, but during the last couple of years, that has changed. The new American pastime has become getting long Apple Inc. stock (NASDAQ:AAPL) any way that you can, and wait for the profits to accumulate. While the above saying may not be absolute true, increasing numbers of investors to AAPL trading, make the current topic about AAPL risk in a certain time frame is indeed worthy to be studied thoroughly.

For a rational financial decision maker, expected returns constitute only one part of the decision making process. Another part that must be taken into

Corresponding author: Zijing Zhang, Department of Mathematics and Statistics, University of Massachusetts Amherst, USA. Email: zzhang@math.umass.edu. consideration is the volatility or risk of returns. Therefore, understanding the risks and volatility involved in stock investing is essential.

It is helpful to know whether there are variations in the risk of stock returns by the day-of-the-week as well as during different seasons. If investors can identify a certain pattern in the risk, then it would be easier to make investment decisions based on both returns and risk. It is also important to know whether a high stock performance is associated with a correspondingly high risk taking behavior. For example, there have been extensive studies of the relation between aggregate volatility and expected returns of the market, see Campbell and Hentschel (1992) [29], Campbell (1996) [28], and Guo and Whitelaw (2003) [30]. Uncovering certain volatility or risk patterns in returns might also benefit investors in option pricing, portfolio optimization, and risk management.

It is well known that the financial institutions with significant amounts of trading activity are vulnerable to extreme market movements. Hence risk quantification, i.e. estimations of probabilities of large losses in financial markets, has become a primary concern for regulators and also for internal risk control. Ideally, 
the best and most informative risk measure of financial vulnerability is given by the whole tail of the loss distribution. A popular method of risk measurement is the Value-at-Risk (VaR for short), which is defined as the loss level that will not be exceeded with a certain confidence level during a certain period of time. The VaR was firstly used as an internal management tool by a number of banks after the 1987 crash,then improved by J.P. Morgan who designed its Risk Metrics System in 1994. It has emerged as one of the most used risk measures in the financial industry, mostly because of its simplicity and intuitive interpretation. Details can be found on the homepage of MSCI. To make the risk measurement coherent, the quantity of Expected Shortfall (ES) is also widely used. The ES of an asset or a portfolio is the average loss, given that VaR has been exceeded. Thus, it is also called conditional value at risk. The advantage of ES is that it is not only sensitive to the shape of the loss distribution in the tail of the distribution, but also possesses the properties required for a coherent risk measure as defined by Artzner (1999) [10].

Hence, the goal of this paper is to characterize the VaR of AAPL relative returns. Based on investigations of the day-of-the-week effect and seasonal effect in extreme event risk, we also provide valuable and applicable analysis for investors who are interested in Apple Inc. stock. The major obstacle to this investigation is a viable measure of tail risk over time. Ideally, one would directly construct a measure of aggregate tail risk dynamics from the time series of stock returns in analogy to dynamic volatility estimated from a GARCH model. But dynamic tail risk estimates are infeasible in a univariate time seriesmodel due to the infrequent nature of extreme events. In this paper, by using the Extreme Value Theory, we not only overcome this problem, but also analyze the week effect as well as the seasonal effect based on our computation of the small quantile (usually $5 \%$ or $1 \%$ ) of VaR. However, we should be aware of various layers of uncertainty in extreme value analysis; for example, the parameter uncertainty, model uncertainty, and data uncertainty. In a sense, it is never possible to have enough data in an extreme value analysis.

Here we first examine certain statistical properties of the time series of stock returns, including stationarity, correlations as well as non-normal distributions. Thereafter, we apply the extreme value analysis on the tested AAPL returns sample set. The calendar effect in stock market returns includes day-of-the-week effect, weekend effect, January effect, and holiday effect, etc. It has been widely studied and investigated in finance literature. Studies by Cross (1973) [1], and Rogalski (1984) [2] demonstrate that there are differences in distribution of stock returns for each day of the week. Studies by Baillie and DeGennaro (1990) [3], Berument and Kiymaz (2001) [4] posit that day-of-the-week effect has an impact on stock market volatility. In recent years, another stream of research has considered seasonality in stock returns and volatility, see Saunders (1993) [5], Bouman and Jacobsen (2002) [6], Hirshleifer and Shumway (2003) [7], Kamstra, Kramer and Levi (2003) [8], and Cao and Wei (2005) [9], etc. These studies generally report that calendar anomalies are present in both returns and volatility equations in the stock market. None of these studies, however, test for the possible existence of day-of-the-week and seasonal variation in stock return VaR.

Empirical findings in this paper show that both the day of the week effect and seasonal effect are present in the AAPL VaR. In the empirical results of the day-of-the-week effect on AAPL tail risk, we observe the lowest VaR of AAPL returns on Fridays and Mondays. We also find that the lower VaR occur on Q1 and Q2 during the test period. AAPL VaR and SPY VaR were compared, the AAPL was found to have its own personality.

In terms of the organization of this paper, we first introduce the dataset and present a comprehensive set of diagnostic checks on it in section 2. We review 
certain aspects of Value-at-Risk and introduce the extreme value approach to $\mathrm{VaR}$ in section 3. We apply the extreme value approach to get the estimation of VaR of the given datasets in section 4. Section 5 contains the empirical tests and results on the AAPL datasets by day-of-the-week. The seasonal effect on AAPL returns VaR and economic implications of the empirical results are examined in section 6 . The comparisons between AAPL returns VaR and SPY returns VaR are discussed in both section 5 and 6 .

\section{Data Exploration and Statistical Analysis}

\section{Data Description}

In this study, we examine the daily AAPL stock price activity over the twenty-year period, July 3, 1995 to July 2, 2015. The collection of AAPL daily adjusted closing price was from Yahoo Finance ${ }^{1}$. The adjusted closing price is used to develop an accurate track record of the stock's performance.

Further, use the negative log return to examine extreme losses of the stock. Let $p_{t}$ denote the adjusted closing price of a stock on day $t$, then the daily percentage change on the day is defined by

$$
r_{t}=-100 \log \frac{p_{t}}{p_{t-1}}=100 \log \frac{p_{t-1}}{p_{t}} .
$$

The reason for using the negative returns is that we are mainly interested in the possibility of large losses rather than large gains.

Fig. 1 shows the time plots of adjusted closing price and negative daily log returns of AAPL stock from July 3, 1995 to July 2, 2015. The upper plot shows that AAPL stock price has skyrocketed over 100 times since 2005. The lower plot shows AAPL negative daily log return time series.

We also observe that there are more pronounced peaks than one would expect from Gaussian data. Table 1 summarizes the basic statistical characteristics of the whole AAPL stock negative daily log return

\footnotetext{
${ }^{1}$ http://finance.yahoo.com/
}

series. Note that the expected AAPL log returns during the test period is 0.09 . The skewness and kurtosis measures are highly significant, and those indicate substantial departures from normality.

In statistics, skewness and kurtosis, which are normalized third and fourth central moments of a process, are often used to summarize the extent of asymmetry and tail thickness. For the normal distribution, kurtosis is 3 .

Since the possibility of time-varying variance and non-normal behavior are noticed, we provide a formal test to check the stationarity and normality of the return process.

\section{Test for Stationary Property}

The invariance of statistical properties of the return process in time corresponds to the stationarity hypothesis that the joint probability distribution of the returns does not change when shifted in time. It is not obvious whether AAPL returns verify this property in calendar time since financial time series data often have non-stationary behaviors, such as trends, and cycles. Here we use the KPSS test [24], to verify the hypothesis of weak stationarity, i.e. time invariance of the mean value and the autocorrelation function of AAPL returns.

Proceeding in the spirit of Kwiatkowski, Phillips, Schmidt and Shin (1992) [24], we assume that the series $\left\{r_{t}\right\}_{t=1}^{T}$ can be decomposed into the sum of a deterministic trend, a random walk and a stationary error. We express this symbolically by writing

$$
r_{t}=\beta t+\alpha_{t}+\varepsilon_{t},
$$

where the constant $\beta$ is the trend; $\varepsilon_{t}$ is assumed to be stationary; and $\alpha_{t}$ is a random walk, i.e.

$$
\alpha_{t}=\alpha_{t-1}+u_{t}
$$

Here $\left\{u_{t}\right\}$ is a white noise series with zero mean and variance $\sigma_{u}^{2}$.

The hypothesis for the KPSS test is

$$
H_{0}: \sigma_{u}^{2}=0 \text { vs } H_{1}: \sigma_{u}^{2} \neq 0 .
$$



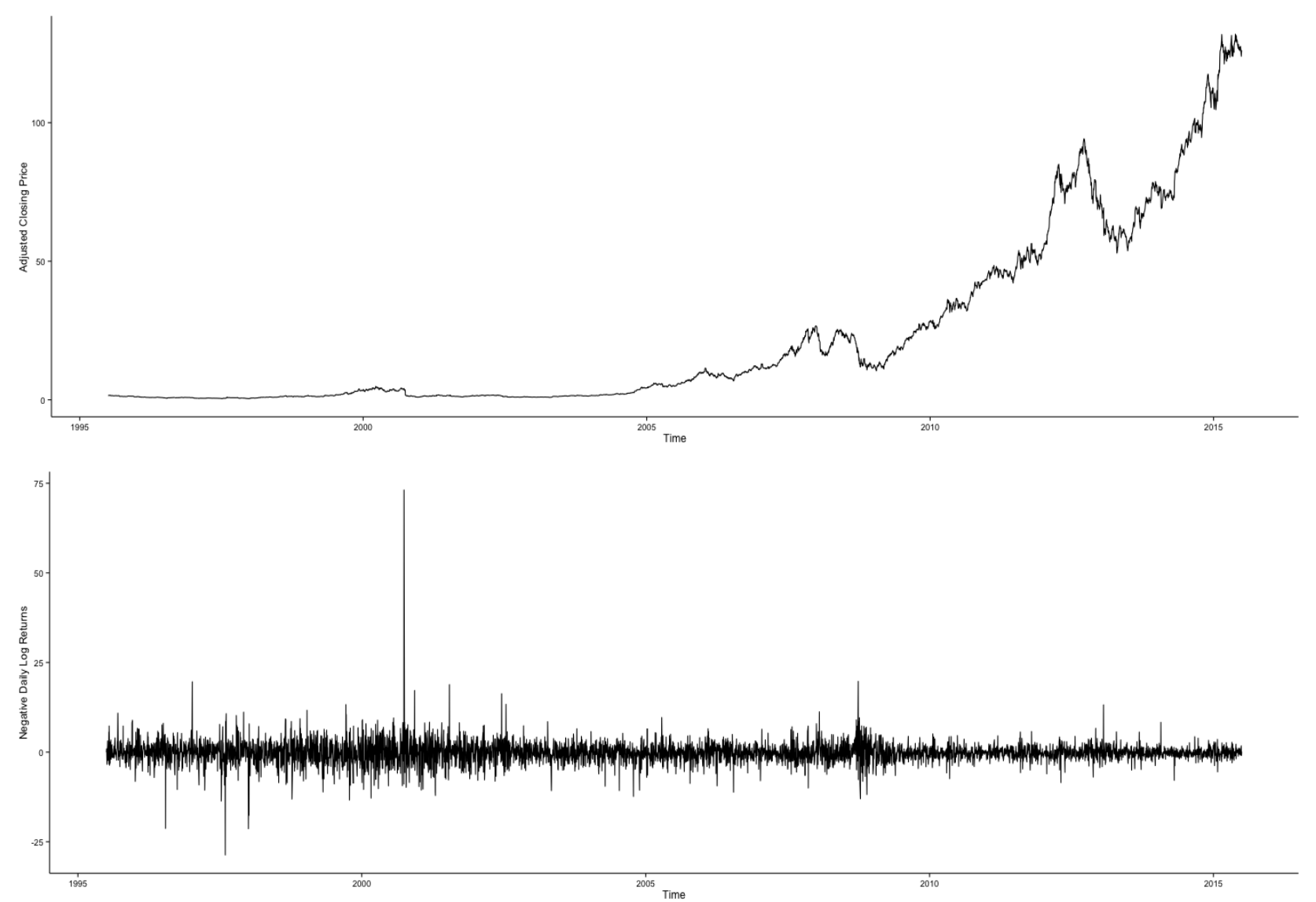

Fig. 1 Time plots of AAPL stock from 1995-07-03 to 2015-07-02. The upper panel is for adjusted closing price, and the lower panel is for negative daily log returns.

Table 1 Summary statistics of the AAPL negative daily log returns July 3, 1995 - July 2, 2015

\begin{tabular}{llllll}
\hline Mean & Range & Std dev & Skewness & Kurtosis & Observations \\
\hline-0.09 & $(-28.69,73.12)$ & 3.05 & 2.55 & 70.82 & 5035 \\
\hline
\end{tabular}

Assume $e_{t}=r_{t}-\left(\hat{\beta} t+\hat{\alpha}_{t}\right)$ as residuals of the regression of $r_{t}$ on an intercept and time trend, $S_{t}=\sum_{i=1}^{t} e_{i}, t=1,2, \cdots, T$, as the partial sum process of the residuals. The KPSS test statistics is

$$
K P S S=\frac{T^{-2} \sum_{t=1}^{T} S_{t}^{2}}{s^{2}}
$$

where $s^{2}$ is a consistent estimator of the long-run variance of $\alpha_{t}$.

The rejection rule is that if the value of the KPSS statistic in Eq.(3) exceeds the critical values estimated in [24], or the $p$-value is less than or equal to the significance level $\alpha$, we reject $H_{0}$.
The results of applying the KPSS test on the AAPL negative daily log returns from July 3, 1995 to July 2, 2015 are shown in Table 2. For the null hypothesis which claims that the series follows a straight line time trend with stationary errors,i.e. $\beta \neq 0$ in Eq.(2), the $p$-value is 0.1 and the corresponding KPSS statistic is 0.080433 . In addition, for the null hypothesis that the series is stationary around a constant rather than a trend with stationary errors, i.e. $\beta=0$ in Eq.(2), the $p$-value is 0.1 and the corresponding KPSS statistic is 0.20611 . In conclusion, the KPSS test result indicates that the AAPL negative daily log returns is stationary from July 3, 1995 to July 2, 2015. 
Table 2 Tests for the AAPL negative daily log returns from July 3, 1995 to July 2, 2015

\begin{tabular}{|c|c|c|c|c|}
\hline & Null Hypothesis $H_{0}$ & Stats & $p$-value & Test Result \\
\hline $\begin{array}{l}\text { KPSS test } \\
\text { for stationary }\end{array}$ & $\begin{array}{c}\text { The series is stationary } \\
\text { around a straight line } \\
\text { time trend } \\
\text { The series is stationary } \\
\text { around a constant. }\end{array}$ & $\begin{array}{l}0.08 \\
0.21\end{array}$ & $\begin{array}{l}0.1 \\
0.1\end{array}$ & $\begin{array}{l}\text { Accept } H_{0} \\
\text { the series is } \\
\text { stationary. }\end{array}$ \\
\hline $\begin{array}{l}\text { Shapiro-Wilk } \\
\text { test for } \\
\text { normality }\end{array}$ & $\begin{array}{c}\text { The series come from } \\
\text { a normally distributed } \\
\text { population. }\end{array}$ & 0.86 & $2.2 \mathrm{e}-16$ & $\begin{array}{l}\text { Reject } H_{0}, \\
\text { the series does } \\
\text { not come from } \\
\text { a normal } \\
\text { distribution. }\end{array}$ \\
\hline $\begin{array}{l}\text { Ljung-Box } \\
\text { test for } \\
\text { correlation }\end{array}$ & $\begin{array}{c}\rho_{1}=\rho_{2}=\cdots=\rho_{5}=0 \\
\rho_{1}=\rho_{2}=\cdots=\rho_{10}=0 \\
\rho_{1}=\rho_{2}=\cdots=\rho_{15}=0\end{array}$ & $\begin{array}{c}16.455 \\
30.34 \\
42.303\end{array}$ & $\begin{array}{l}0.005658 \\
0.0007536 \\
0.0002018\end{array}$ & $\begin{array}{l}\text { Reject } H_{0}, \\
\text { the series is not } \\
\text { autocorrelated. }\end{array}$ \\
\hline
\end{tabular}

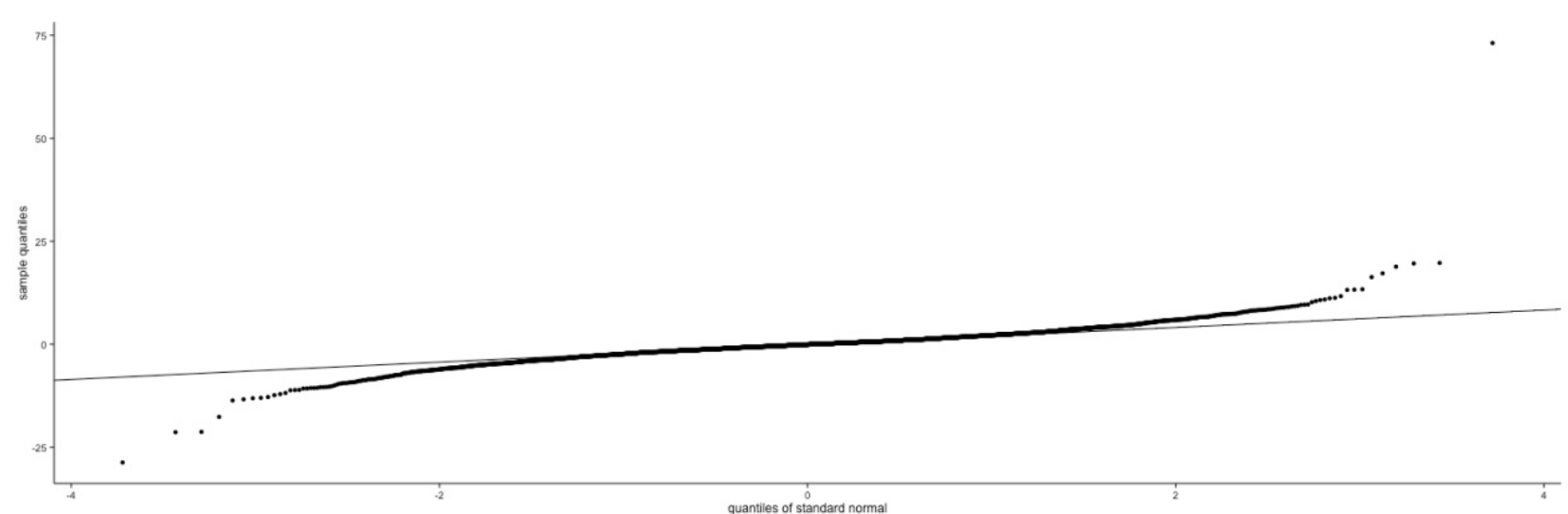

Fig. 2 Quantile-quantile plot of AAPL negative log returns from 1995-07-03 to 2015-07-02 against the normal distribution.

\section{Test for Normality}

In studying the financial time series, one common assumption is that the process follows normal distribution. However, it is barely true in the real stock return series. Our study shows that the AAPL stock returns are not normally distributed.

We begin by forming a QQ-plot of the AAPL negative daily log returns sample set against the normal distribution, in order to confirm that an assumption of normality is unrealistic, and that the innovation process has fat tails or is leptokurtic - see Fig.2.

We also use one of the most powerful formal normality tests ${ }^{2}$, the Shapiro-Wilk test [25], to verify an empirical fact that the AAPL stock returns do not have the normality property.

${ }^{2}$ Razali and Wah (2011) demonstrate it in [26].
The Shapiro-Wilk test utilizes the null hypothesis principle to check whether the series $\left\{r_{t}\right\}_{t=1}^{T}$ comes from a normally distributed population. The Shapiro-Wilk test statistic is defined as

$$
W=\frac{\left(\sum_{t=1}^{T} a_{t} r_{t}\right)^{2}}{\sum_{t=1}^{T}\left(r_{t}-\bar{r}\right)^{2}},
$$

where $r_{t}$ is the t-th order statistic; $\bar{r}$ is the sample mean; $\left(a_{1}, a_{2}, \cdots, a_{T}\right)$ are the weights. $\left(a_{1}, a_{2}, \cdots, a_{T}\right)=\frac{m^{T} V^{-1}}{\left(m^{T} V^{-1} V^{-1} m\right)^{1 / 2}}$ $m=\left(m_{1}, m_{2}, \cdots, m_{n}\right)^{T}, \quad m_{1}, m_{2}, \cdots, m_{n}$ are the expected values of the order statistics of independent and identically distributed random variables sampled from the standard normal distribution, and $V$ is the covariance matrix of those order statistics. 
The value of $W$ lies between zero and one. Small values of $W$ lead to the rejection of normality whereas a value of one indicates the normality of data. We reject the null hypothesis if the $p$-value of the test is less than the predetermined significance level.

Applying the Shapiro-Wilk test on the AAPL negative daily log returns, we get the Shapiro-Wilk statistic $W=0.86$. The $p$-value is less than $2.2 e$ -16 , see Table 2 . Hence, we reject the null hypothesis at the significant level $1 \%$ and conclude that the AAPL returns are not normally distributed during above time period.

\section{Test for Correlations}

In the finance literature, testing for zero autocorrelations has been used as a tool to verify the efficiency of the market hypothesis. Since applying extreme value theory on a data set suggests that the time series are highly uncorrelated with a commoncumulative distribution function, we need to check the correlations of the AAPL returns.

We begin by considering the autocorrelation function of a time series $\left\{r_{t}\right\}$. The correlation between $r_{t}$ and its past values $r_{t-l}$ is called the lag$l$ autocorrelation of $\left\{r_{t}\right\}$ and is commonly denoted by $\rho_{l}$. Under the weakly stationary assumption, we assume $\rho_{l}$ is a function of $l$ only, i.e.

$$
\rho_{l}=\frac{\operatorname{Cov}\left(r_{t}, r_{t-l}\right)}{\sqrt{\operatorname{Var}\left(r_{t}\right) \operatorname{Var}\left(r_{t-l}\right)}}=\frac{\operatorname{Cov}\left(r_{l+1}, r_{1}\right)}{\operatorname{Var}\left(r_{1}\right)}=\frac{\gamma_{l}}{\gamma_{0}},
$$

where the property $\operatorname{Var}\left(r_{t}\right)=\operatorname{Var}\left(r_{1}\right)=\gamma_{0}$ for a weakly stationary series is used.

For a given sample of returns $\left\{r_{t}\right\}_{t=1}^{T}$, let $\bar{r}=\left(\sum_{t=1}^{T} r_{t}\right) / T$ is the sample mean. The lag- $l$ sample autocorrelation of $\left\{r_{t}\right\}$ can be represented as:

$$
\begin{gathered}
\hat{\rho}_{l}=\frac{\sum_{t=l+1}^{T}\left(r_{t}-\bar{r}\right)\left(r_{t-l}-\bar{r}\right) /(T-l+1)}{\sum_{t=1}^{T}\left(r_{t}-\bar{r}\right)^{2} /(T-1)}, \\
0 \leq l \leq T-1 .
\end{gathered}
$$

If a time series is not autocorrelated, then estimates of $\hat{\rho}_{l}$ will not be significantly different from 0 .

Fig.5 shows the sample autocorrelation coefficient $\hat{\rho}_{l}$ plotted against different lags $l$ (measured in days), along with the $95 \%$ confidence band around zero for AAPL negative daily log returns, for the period July 3, 1995 to July 2, 2015. The dashed lines represent the upper and lower $95 \%$ confidence bands $\pm \frac{1.96}{\sqrt{T}}$, where the time length for our AAPL returns is $T=5036$ days. Fig. 5 shows a small autocorrelation in AAPL daily log price changes. Even in the cases where the autocorrelations are outside the confidence bands, the autocorrelation coefficients are quite small, less than $5 \%$.

Besides using the graphical plot to check autocorrelation, we also apply a formal statistic test: the Ljung-Box test by Ljung and Box (1978) [27],

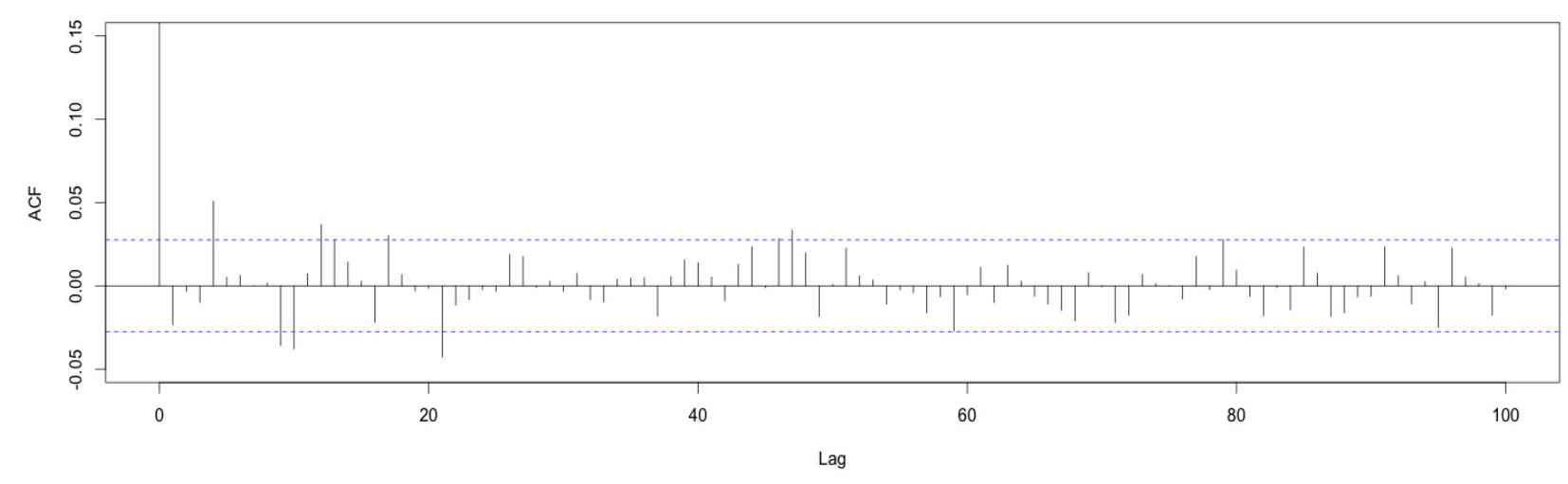

Fig. 3 Sample autocorrelation coefficients up to 100 lags for AAPL returns from 1995-07-03 to 2015-07-02. 
which checks serial correlation of the time series. The null and alternative hypothesis of the Ljung-Box test is

$$
\begin{gathered}
H_{0}: \rho_{1}=\cdots=\rho_{m}=0 \\
v s \\
H_{1}: \rho_{i} \neq 0 \text { for } i \in\{1,2, \cdots, m\}
\end{gathered}
$$

As Ljung and Box [27] proposed, under the assumption that $\left\{r_{t}\right\}_{t=1}^{T}$ is an i.i.d. sequence with certain moment conditions, the modified Portmanteau statistic is defined as

$$
Q(m)=T(T+2) \sum_{l=1}^{m} \frac{\hat{\rho}_{l}^{2}}{T-l} .
$$

It is asymptotically a chi-squared random variable with $m$ degrees of freedom, i.e. $Q(m) \sim \chi_{m}^{2}$ under the null hypothesis $H_{0}$. In the definition of $Q(m), T$ is the sample size, $\hat{\rho}_{l}$ is the sample autocorrelation at lag $l$, and $m$ is the number of lags being tested.

The decision rule is to reject $H_{0}$ if $Q(m)>\chi_{1-\alpha, m}^{2}$ for significance level $\alpha$, where $\chi_{1-\alpha, m}^{2}$ denotes the $100(1-\alpha)$ th percentile of a chi-squared distribution with $m$ degrees of freedom. Also, one should reject $H_{0}$ if the $p$-value of $Q(m)$ is less than or equal to the significance level $\alpha$.

The test result in Table 2 confirms that the AAPL returns series does not have strong serial correlations during the test period. The $p$-values of lag 5, lag 10 and lag 15 Ljung-Box test for AAPL returns are all less than significant level $1 \%$.

Based on the statistical analysis for AAPL negative daily log returns, we discovered that the AAPL returns is a stationary, and uncorrelated time series, yet is not normally distributed. Some computations of VaR are based on the assumption that the series $\left\{r_{t}\right\}$ is normally distributed, or has $t$-distribution, see reference [15] [32] [33] [34] [35]. That is the main reason why these study can use volatility to estimate
VaR. However, the real time series $\left\{r_{t}\right\}$ may not follow any known distributions, such as the normal or $t$-distribution. To overcome the difficulty of $\left\{r_{t}\right\}$ having an unknown distribution, we compute the VaR of AAPL returns by applying Extreme Value Theory, which avoid making assumptions of the distribution of $\left\{r_{t}\right\}$.

\section{Methodology}

While exposure to risk can be summarized as a single number by estimating the $\mathrm{VaR}$, which is defined by Jorion [11] as "the worst expected loss over a great horizon within a given confidence level", it is crucial to have an accurate estimate on VaR. Following the approach by Longin (1999a, b) [12] [13], and Ruey S. Tsay [14], we introduce the statistical principles behind VaR as well as the VaR estimation methodology in this section.

\subsection{VaR of a time series}

$\mathrm{VaR}$ is the amount that might be lost in a portfolio of assets over a specified time period $T$ with a specified small failure probability $\alpha$, usually set as 0.01 or 0.05 . Suppose a random variable $X$ characterizes the distribution of negative returns of a portfolio over a certain time horizon $T$, the right-tail $\alpha$-quantile of the portfolio is then defined to be the $\mathrm{VaR}_{\alpha}$ such that

$$
\operatorname{Pr}\left(X \leq \mathrm{VaR}_{\alpha}\right)=1-\alpha .
$$

The $\mathrm{VaR}_{\alpha}$ is the largest value for $X$ such that the probability of a loss over the time horizon $T$ is no more than $1-\alpha$. Although the parameters $T$ and $\alpha$ are arbitrarily chosen, the analysis in this study does not refer to the process of choosing the two parameters of VaR which were considered to be $T=1$ day, $\alpha \in\{0.01,0.05,0.1\}$.

The crux of being able to provide an accurate estimate for $\mathrm{VaR}$ is in estimating the cutoff return $\mathrm{VaR}_{\alpha}$. Studies of VaR are essentially concerned with the estimation of the cumulative distribution function 
(CDF) of portfolio negative returns and/or its quantile, especially the upper tail behavior of the loss CDF. Therefore, the CDF of $\left\{X_{t}\right\}$ is the focus of econometric modeling. Different methods for estimating the CDF give rise to different approaches to VaR estimation.

\subsection{Extreme Value Theory approach to Value-at-Risk}

In this paper, we further estimated the upper tail behavior of the AAPL returns CDF by using the extreme value approach. Extreme Value Theory (EVT) is experiencing a boom in the financial field, especially with respect to its application to the market risk. Its appearance as a popular instrument for estimating VaR can be explained as a consequence of two factors. On the one hand, the assumption of the normality of financial markets does not reflect the reality of the situation. As a consequence, the VaR estimation methods which are based on the normality assumption underestimates the risk. Historical or Monte Carlo simulation methods arise as alternative methods. But given the difficulties and the inefficiencies of these methods, EVT is sought out as a new solution.

The mathematical foundation of EVT is based on the class of extreme value limit theories, originally posited by Fisher and Tippett (1928) [16] and later derived rigorously by Gnedenko (1943) [17]. The central result in EVT is that the extreme tail of a wide range of distributions can approximately be described by the Generalized Pareto distribution (GPD), which is derived by Smith (1989) [18], Davison and Smith (1990) [21].

For a random variable $X$, we first fix some high threshold $\mu$ and consider the distribution of excess values $Y=X-\mu$, which is defined as:

$$
\begin{aligned}
& F_{\mu}(y)=\operatorname{Pr}(X-\mu \leq y \mid X>\mu) \\
& =\frac{F(\mu+y)-F(\mu)}{1-F(\mu)},
\end{aligned}
$$

where $F$ is the underlying distribution of $X, F_{\mu}$ is the conditional excess distribution function. In fact, Pickands (1975) [22] introduced the GPD as a two parameter family of distributions for exceedance over a threshold.

Extreme Value Theory. Assume $\left\{X_{t}\right\}$ is a sequence of stationary, uncorrelated random variables with distribution $F$. For any $\mu>0$, let $F_{\mu}$ be the conditional excess distribution function, for random variables defined in (9) with $Y_{t}=X_{t}-\mu$. Let $\omega_{F}=\sup \{x: F(x)<1\}$, then

$$
\lim _{\mu \rightarrow \omega_{F}} F_{\mu}(y)=H_{\sigma_{\mu}, \xi}(y)
$$

where $H_{\sigma_{\mu}, \xi}(y)$ is called GPD, specified as

$$
H_{\sigma_{\mu}, \xi}(y)=1-\left(1+\xi \frac{y}{\sigma_{\mu}}\right)_{+}^{-1 / \xi}
$$

The parameters of GPD are the scale parameter $\sigma_{\mu}$ and the shape parameter $\xi$.

Although we may not know the distribution of each individual random variable $X_{t}$, EVT specifically describes the tail distribution. The tail fatness of a distribution is reflected by the shape parameter:

$\xi<0$ refers to thin tails;

$\xi=0$ implies that the kurtosis is 3 as for a standard normal distribution;

$\xi>0$ implies fat tails.

Therefore, the shape parameter measures the speed with which the distribution's tail approaches zero. The fatter the tail, the slower the speed and the higher the shape parameter. Using GPD, EVT models the right tail of the distribution, i.e. the returns in excess of a threshold. Because we are interested in extreme loss, the EVT analysis is developed on negative stock returns. As tested in section 2 that AAPL returns are stationary and serially uncorrelated, its $\mathrm{VaR}$ is analyzed by using EVT.

In the literature, an optimal threshold is selected by employing graphical methods, the mean excess plot ${ }^{3}$

\footnotetext{
${ }^{3}$ Details about the mean excess plot are described in Davison and Smith (1990)[31].
} 
and Hill plot ${ }^{4}$. The mean excess plot for threshold exceedance is a diagnostic plot drawn before fitting any model and can therefore give guidance about what threshold to use. One difficulty with this method is that the sample mean excess plot typically shows very high variability, particularly at high thresholds. This can make it difficult to decide whether an observed departure from linearity is in fact due to the failure of the GPD or is just sample variability. As an alternative approach to choose threshold, the Hill plot has some advantages. It displays the estimated values of the shape parameter $\xi$ as a function of the cut-off threshold, so that one can easily find some interval of candidate cut-off points that yields stable estimates of the shape parameter $\xi$. Here we use both approaches to choose a reasonable threshold, see Figure 4.

According to the research of Hosking and Wallis (1987) [20], for the shape parameter $\xi>-0.5$, it is shown that maximum likelihood regularity conditions are fulfilled and that maximum likelihood estimates $\left\{\hat{\xi}_{n},\left(\hat{\sigma}_{\mu}\right)_{n}\right\}$ based on a sample of $n$ excesses are asymptotically normally distributed. Therefore, we choose to use the parametric approach, maximum likelihood method (MLE) to estimate the two parameter in GPD, which are the shape parameter $\xi$ and the location parameter $\sigma_{\mu}$.

Next, we make explicit the relationship between excess value and a observed return series $\left\{r_{t}\right\}$. Assume that $\left\{r_{t}\right\}$ have distribution $F$, and a high enough threshold $\mu$ is given. We define the number of exceedance of the threshold $\mu$ within $\left\{r_{1}, \cdots, r_{n}\right\}$ as:

$$
N_{\mu}=\operatorname{card}\left\{t: r_{t}>\mu, t=1, \cdots, n\right\} .
$$

Then the conditional excess distribution function can be presented as:

$$
F_{\mu}(y)=\operatorname{Pr}\left(r_{t}-\mu \leq y \mid r_{t}>\mu\right)=\frac{F(\mu+y)-F(\mu)}{1-F(\mu)} .
$$

\footnotetext{
${ }^{4}$ Technical details about Hillplot can be found in Hill (1975)[19].
}

Denote $\bar{F}_{\mu}(y)=1-F_{\mu}(y)$, then

$$
\bar{F}_{\mu}(y)=\operatorname{Pr}\left(r_{t}-\mu>y \mid r_{t}>\mu\right)=\frac{\bar{F}(\mu+y)}{\bar{F}(\mu)},
$$

which is equivalent to

$$
\bar{F}(\mu+y)=\bar{F}(u) \bar{F}_{\mu}(y) .
$$

Consequently, the estimators of $\bar{F}(u)$ and $\bar{F}_{\mu}(y)$ can be written as:

$$
\begin{aligned}
& \widehat{\bar{F}(u)}:=\frac{1}{n} \sum_{i=1}^{n} I\left(X_{i}>\mu\right)=\frac{N_{\mu}}{n}, \\
& \widehat{\bar{F}_{\mu}(y)}:=1-H_{\hat{\sigma}_{\mu}, \hat{\xi}}(y)=\left(1+\hat{\xi} \frac{y}{\hat{\sigma}_{\mu}}\right)_{+}^{-1 / \hat{\xi}},
\end{aligned}
$$

where $\xi$ and $\hat{\sigma}_{\mu}$ are (maximum likelihood) estimators of the shape parameter $\xi$ and location parameter $\sigma_{\mu}$. Therefore the tail estimator can be written as:

$$
\overline{\bar{F}(\mu+y)}=\frac{N_{\mu}}{n}\left(1+\hat{\xi} \frac{y}{\hat{\sigma}_{\mu}}\right)_{+}^{-1 / \hat{\xi}} .
$$

This relationship between probabilities allows us to obtain VaR for the original asset return series $\left\{r_{t}\right\}$. More precisely, for a specified small probability $\alpha$

$$
\alpha=\operatorname{Pr}\left(r_{t}>\mu+y\right)=\bar{F}(\mu+y),
$$

where the $\alpha$-th upper tail quantile VaR of $\left\{r_{t}\right\}$ is $\mu+y$. Consequently, for a given small probability $\alpha$, one can check that the VaR of holding a long position in the asset underlying return $\left\{r_{t}\right\}$ is

$$
\mathrm{VaR}_{\alpha}=\left\{\begin{array}{c}
\mu+\frac{\hat{\sigma}_{\mu}}{\hat{\xi}}\left(\left(\frac{n}{N_{\mu}} \alpha\right)^{-\hat{\xi}}-1\right), \quad \hat{\xi} \neq 0 \\
\mu+\hat{\xi} \ln \left(\frac{n \alpha}{N_{\mu}}\right), \quad \hat{\xi}=0
\end{array}\right.
$$

We preferred to use the extreme value approach, or named GPD approach in this study to tail estimation mainly for three reasons. One is that in finite samples of the order of points from typical return distributions, 
EVT quantile estimators are more efficient than the historical simulation method. Second, considering the fact that most financial returns series are asymmetric, the EVT approach is advantageous over models which assume symmetric distributions such as $t$ -distributions, or generalized error distribution. Third, comparing with Hill method which is designed specifically for the heavy tail $(\xi>0)$ data, the EVT approach to VaR has larger applicability since it also applicable to light tail $(\xi=0)$ cases or even short tail $(\xi<0)$ cases.

\section{VaR analysis of AAPL and SPY}

In this section, one-day-ahead VaR forecasts are adopted along with the $5 \%, 1 \%$ and $0.1 \%$ level of significance in the empirical investigation. In order to make a comparison, we use AAPL as well as SPDR S\&P 500 ETF (AMEX:SPY) daily negative log returns to compute the VaR and related statistical properties. The daily AAPL negative log returns data set is introduced in section 2.1. The SPDR S\&P 500 ETF is the first and most popular ETF in the U.S.. It tracks one of the most popular indexes in the world, the S\&P 500 Index. The objective of the SPY is to duplicate as closely as possible, before expenses, the total return of the S\&P 500 Index. Since the performance of SPY is thought to be representative of the stock market as a whole, we compare VaR between AAPL returns and SPY returns to find the characteristics of AAPL. The daily SPY negative log returns are examined for the period of July 3, 1995 to July 2, 2015, which is the same test period as AAPL returns. We also ran Shapiro-Wilk test and Ljung-Box test in SPY returns and found no evidence against the non-normal and non-correlated assumptions for the series.

Before applying the extreme value approach to VaR on our data sets, it is necessary to choose a specific threshold, confining the estimation to those observations that are above the given threshold. As mentioned in section 3, we chose the threshold through graphical procedures: Mean Excess plot and Hill plot.

Fig.4 shows the Hill plots and Mean Excess plots, with $95 \%$ confidence bands, for the AAPL returns and SPY returns respectively. Since for the generalized pareto distribution, a possible choice of threshold is given by the value, above which the empirical mean excess value is approximately linear. The right-hand plots of Fig.4 indicate a reasonable choice for AAPL returns where threshold should around 5, and SPY returns threshold should around 2. The Hill estimator estimates the shape parameter $\xi$ in the GPD model as a function of the $N_{\mu}$ exceedances upper order statistics in the return sample. The estimate is taken in the $N_{\mu}$-region where the plot does not change much. For the AAPL returns, $N_{\mu}=280$, with corresponding threshold $\mu=4.153575$; for SPY returns, $N_{\mu}=268$, with corresponding threshold $\mu=1.911077$ would be reasonable.

Table 3 contains the empirical results on the AAPL and SPY daily negative log returns for the whole sample period using a total of 5035 observations. The upper part of Table 3 contains the threshold values and the corresponding exceedances values as well as the maximum likelihood GPD parameter estimates used in the construction of tail estimators of AAPL and SPY negative daily log returnsfrom July 3, 1995 to July 2, 2015. The shape parameter estimates of the right tail are 0.2619561 and 0.2411802 for AAPL and SPY returns, respectively, which indicate that the AAPL returns show fatter tails than the SPY returns. Those values and estimators enable us to estimate the upper $5 \%, 1 \%$ and $0.1 \%$ quantile of the AAPL and SPY negative daily price changes. As is obvious from the estimation of the quantile by means of extreme value theory in this table, the AAPL returns VaR are much larger, even more than two times, than SPY returns VaR. Therefore, AAPL exhibits a more downside risk than SPY.

In order to visualize the model (12) accuracy, we backtest the extreme value approach on the AAPL and 

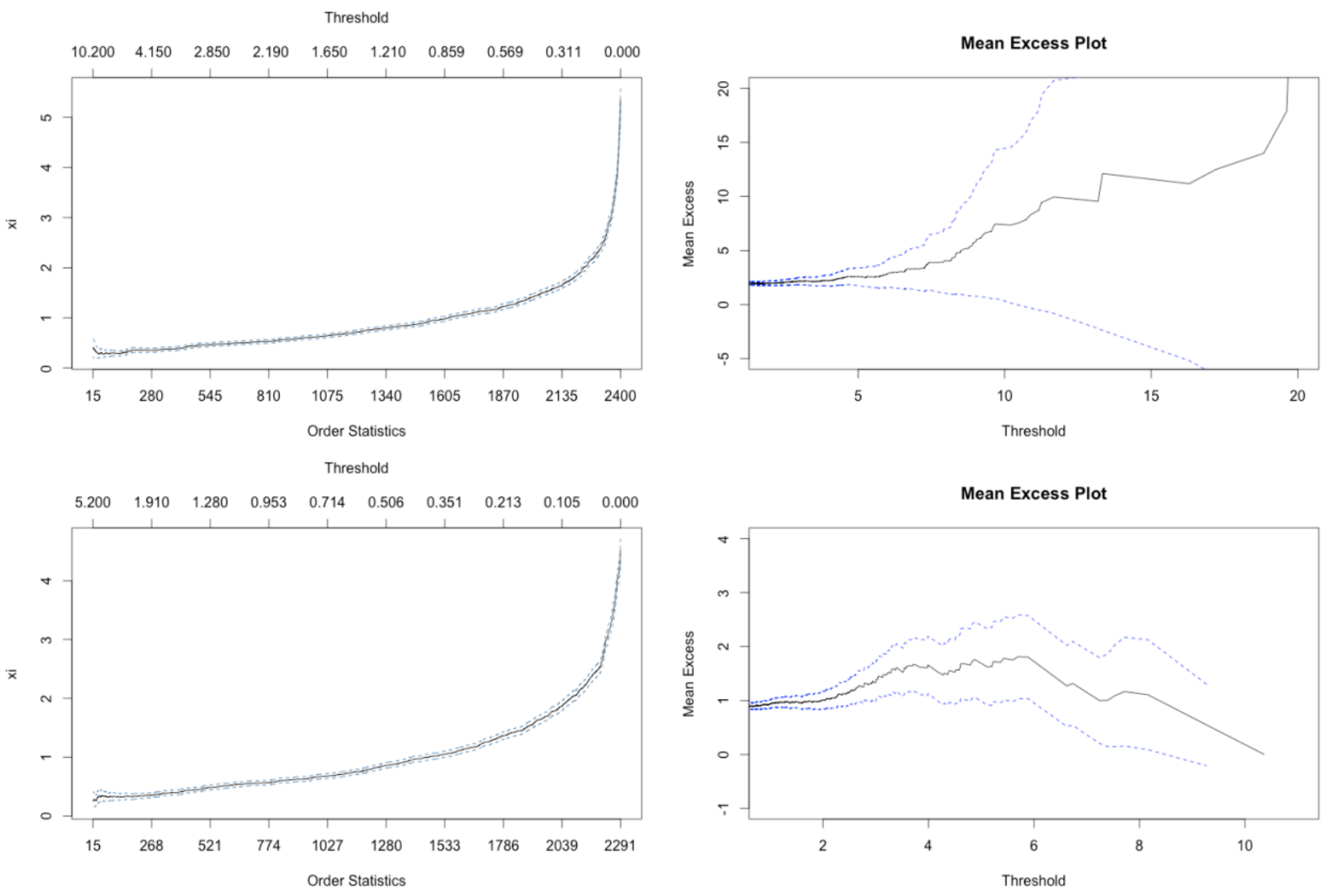

Fig. 4 The left-hand plots are Hill plots for the AAPL returns (top) and SPY returns (lower) with 95\% asymptotic confidence bounds (dotted line) based on the normal asymptotics of the estimator, depending on different threshold values $\mu$. The right-hand plots are Mean excess over plots for AAPL returns (top) and SPY returns (lower) with 95\% Monte Carlo confidence bands.

Table 3 Maximum likelihood GPD parameter estimates and one-day-ahead VaR forecasts with the 5\%, $1 \%$ and $0.1 \%$ level of significance for AAPL and SPY returns.

\begin{tabular}{|l|cc|}
\hline Negative daily log returns (1995-7-3 to 2015-7-2) & AAPL & , SPY \\
\hline Threshold $\mu$ & 4.153575 & 1.911077 \\
Exceedances $N_{\mu}$ & 280 & 268 \\
Shape parameter ML estimator $\hat{\xi}$ & 0.2619561 & 0.2411802 \\
Scale parameter ML estimator $\hat{\sigma}_{\mu}$ & 1.6145412 & 0.7537094 \\
\hline $\operatorname{VaR}(T=1$ day, $\alpha=5 \%)$, & 4.327371 & 1.958427 \\
$\operatorname{VaR}(T=1$ day, $\alpha=1 \%)$ & 7.65061 & 3.463027 \\
$\operatorname{VaR}(T=1$ day, $\alpha=0.1 \%)$ & 15.64864 & 6.935867 \\
\hline
\end{tabular}

SPY returns and show the fitness summary in Fig.5 and Fig.6. At the top panel of Fig.5 and Fig.6, the probability density function of the empirical distribution and the log probability density function of the empirical distribution are all plotted along with the estimated GPD. The scatterplot and QQ-plot of residuals are at the lower panel. Based on those plots, we find that the estimates fit the given AAPL and SPY returns quite well, even in the far end tail. It confirms that the assumption of an underlying heavy tailed distribution is well in line with the data. In this context, the corresponding estimate of the upper $5 \%, 1 \%$ and $0.1 \%$ quantile of the VaR seems very plausible. 

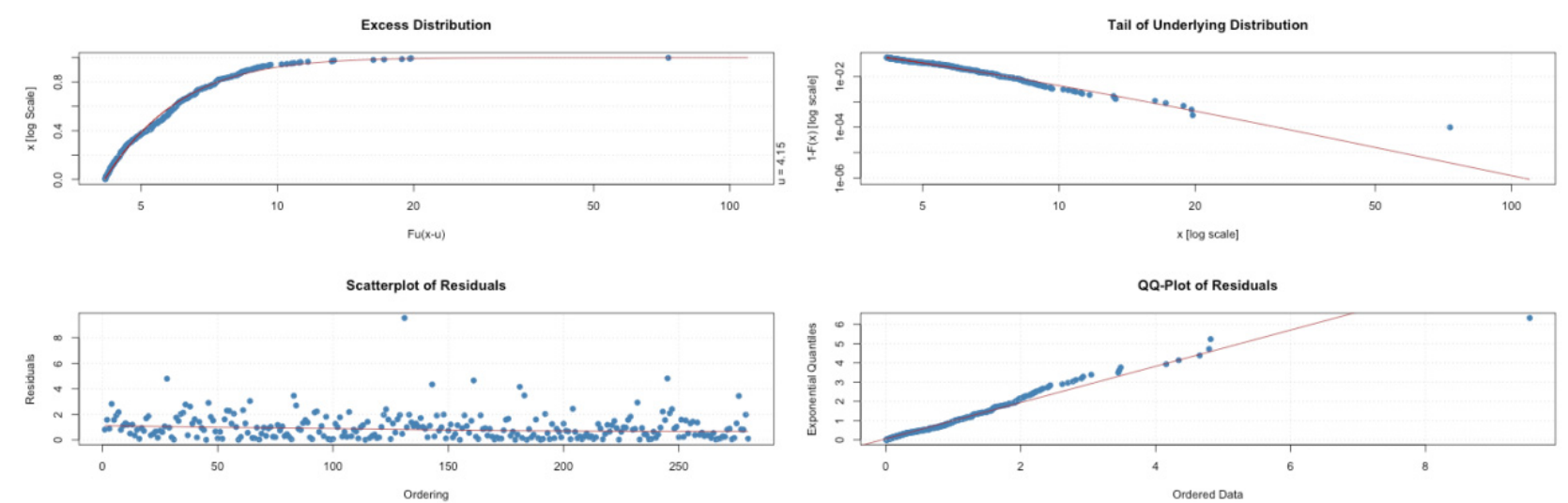

Fig. 5 GPD tail estimates fitness summary of the AAPL returns distributions and the innovations distributions. The points show the empirical distribution of the returns (top) and residuals (lower) and the solid lines (top) represent the tail estimates.
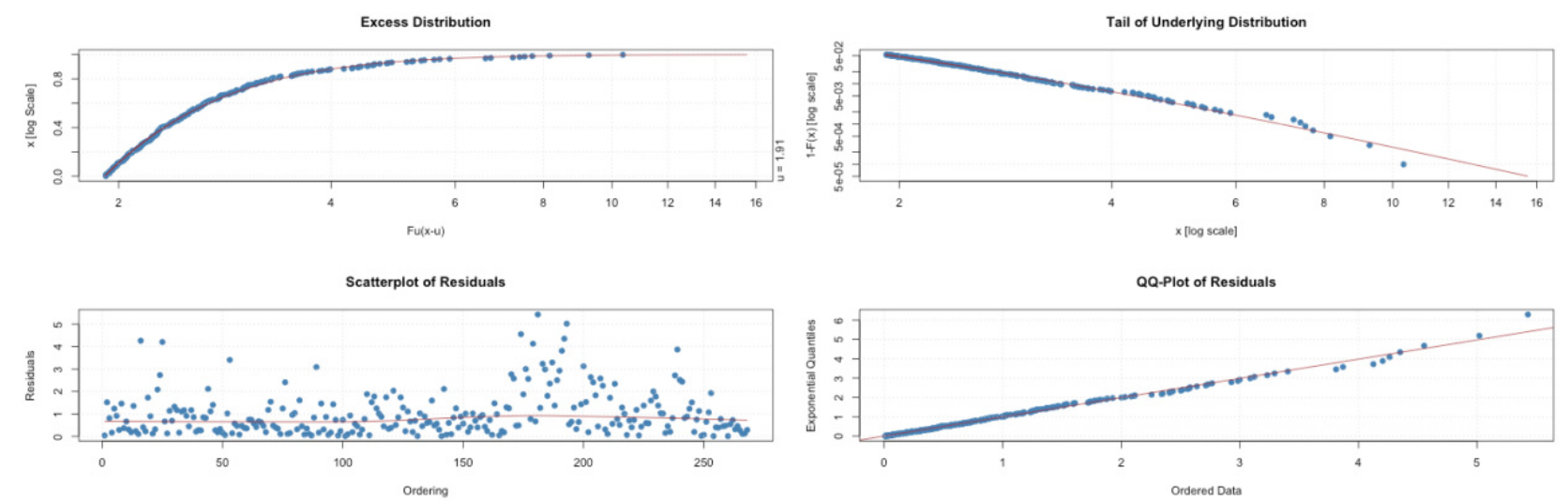

Fig. 6 Diagnostic plots for GPD fit to daily negative log returns of SPY from July 3, 1995 to July 2, 2015.

After modeling the distribution of AAPL and SPY returns and computing all the necessary quantiles, we proceed to the determination of VaR. Using a 2520 day (approximately 10 years) rolling window, we apply an iterative procedure of the EVT based model (12) to predict the 1 -day ahead, $5 \%, 1 \%$ and $0.1 \%$ VaR for the period July 3, 2005 to July 2, 2015. The moving window design starts with the estimation of the VaR model using in-sample period data to predict the 1-day ahead VaR estimate. Then, we move the in-sample period forward by one period to iterate the estimation and prediction. The whole process keeps running forward step by step until the end of the entire data set. Before applying the procedure, we choose the corresponding 95-th sample quantile as the threshold of each in-sample period data. This yields a total of 2516 out-of-sample VaR forecasts for AAPL and SPY returns, respectively. The results obtained for $\mathrm{VaR}$ along with the negative log returns of the AAPL and SPY are shown in the following figures.

From Fig.7, we find that the AAPL returns VaR slightly increased during the financial crisis of 2008. During the middle of 2010 to 2013, it had a decreasing trend. Since then, the AAPL returns VaR seems stable. If we take SPY returns VaR as a comparison, except the increasing during the financial crisis of 2008, we can see that it has been stable for the last decade.

\section{Day-of-the-week Effect on AAPL Value-at-Risk}

As introduced in section 2, two decades are covered for our sample set, 5036 trading days and 5035 AAPL returns in total. Notice that the definition of VaR is based on the upper tail of a loss function. The reason 

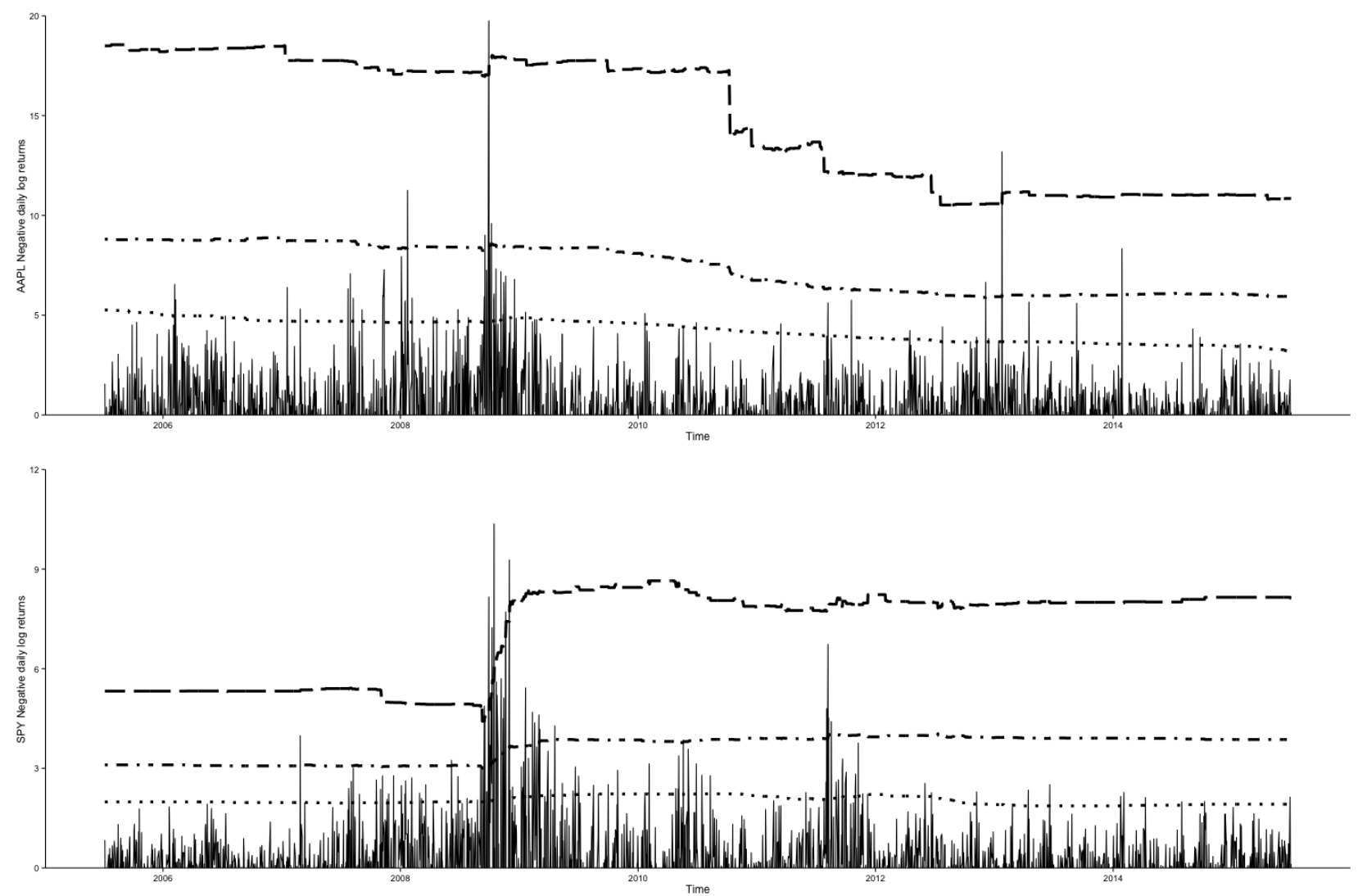

Fig. 7 1-day-ahead, $5 \%$ (dotted), $1 \%$ (dotdash) and $0.1 \%$ (longdash) VaR of AAPL (upper) and SPY (lower) negative daily log returns from July 6, 2005 to July 2, 2015.

we use the negative returns is that loss occurs when the returns are negative for a long financial position. We write the whole sample set as $\left\{r_{t}\right\}=\{$ AAPL Negative daily log returns from July 3, 1995 to July 2, $2015\}$.

To formally test the timing and existence of weekly patterns, we divide the whole data set $\left\{r_{t}\right\}$ to five subsets by day-of-the-week, which is written as:

$$
\left\{d_{i t} r_{t}\right\}, \quad i=1,2,3,4,5
$$

where $d_{i t}$ are dummy variables such that if day $t$ is a Monday $d_{1 t}=1$, if $d_{1 t}=0$ remove the data; if day $t$ is a Tuesday $d_{2 t}=1$, if $d_{2 t}=0$ remove the data, etc. The five subsets are the AAPL return time series for Monday through Friday respectively. The basic statistical characteristics of the five return series are calculated and shown in Table 4.
The AAPL mean returns is calculated to observe differences of expected returns during the week. The hypothesis of equal expected returns for each trading day of the week is rejected for the testing period. Our results show that the highest returns occur on Mondays and the lowest returns occur on Fridays, which have a negative average return. The standard deviation indicates that the trading risk of Fridays is the highest among that of the week. There is less fluctuation in Mondays and Tuesdays return, but there are large fluctuations in Fridays return. The table also reports skewness and kurtosis for the return series of each weekday. The distribution of Mondays and Fridays return are positively skewed while the distribution of all other sample return are negatively skewed, indicating that they are nonsymmetric. Furthermore, Fridays and Wednesdays return exhibit high levels of kurtosis, indicating that these distributions have thicker tails 
Table 4 Summary statistics of the AAPL negative daily log returns by day-of-the-week.

\begin{tabular}{lllllll}
\hline & Mean & Range & Std dev & Skewness & Kurtosis & Obs \\
\hline Mon. & -0.20 & $(-13.02,19.75)$ & 2.89 & 0.51 & 5.78 & 949 \\
Tue. & -0.08 & $(-17.64,13.25)$ & 2.82 & -0.17 & 3.18 & 1032 \\
Wed. & -0.12 & $(-28.69,18.84)$ & 2.98 & -0.26 & 12.17 & 1034 \\
Thu. & -0.14 & $(-21.27,13.19)$ & 3.03 & -0.54 & 4.52 & 1012 \\
Fri. & 0.10 & $(-21.36,73.12)$ & 3.47 & 8.91 & 194.73 & 1008 \\
\hline
\end{tabular}

Table 5 Normality and independent test $p$-value of the AAPL returns by day-of-the-week.

\begin{tabular}{lllll}
\hline & $\mathrm{W}$ & $\mathrm{Q}(5)$ & $\mathrm{Q}(10)$ & $\mathrm{Q}(15)$ \\
\hline Mon. & $<2.2 \mathrm{e}-16$ & 0.2963 & 0.5304 & 0.2641 \\
Tue. & $2.713 \mathrm{e}-15$ & 0.7911 & 0.08139 & 0.1224 \\
Wed. & $<2.2 \mathrm{e}-16$ & 0.8355 & 0.7908 & 0.5826 \\
Thu. & $<2.2 \mathrm{e}-16$ & 0.5063 & 0.8421 & 0.2699 \\
Fri. & $<2.2 \mathrm{e}-16$ & 0.06514 & 0.1182 & 0.08148 \\
\hline
\end{tabular}

than a normal distribution. These initial findings show that the day-of-the-week returns are not normally distributed, they are skewed and leptokurtic. Moreover, on average, an investor buying stock on Friday afternoon and then sell it by Monday afternoon may make more profit.

Next, we examine the normality and independency of the day-of-the week return series. Table 5 reports $p$-values of the Shapiro-Wilk test and the Ljung-Box $Q$ statistics for the AAPL returns at 5-, 10- and 15day lags. We use the Shapiro-Wilk test to test the normality of every subset. $W$ here is the Shapiro-Wilk test statistic in Eq.(4). The normality test result shows that the $p$-value of every weekday subset is far less than significant level 1\%. It indicates that none of these AAPL return subsets has normal behavior during the test period. For all series, the Ljung-Box test is applied to test the serial correlation. $Q(m)$ in the table is the Portmanteau statistic in Eq.(7). The Ljung-Box test result confirms that none of the AAPL return subsets has serial correlations. Rather than using a single value for lag $m$, we choose three different lags $m=5,10,15$ to test the correlation of each series. Even the minimum $p$-value of each series is greater than the usual significant level 5\%. Therefore, we can approximately view all AAPL day-of-the-week return series as stationary, independent, non-normal distributed series. Based on the above tests, in order to test the weekly effects on AAPL VaR, we apply the VaR estimation approach introduced in section 2 to capture the day-of-the-week effect on AAPL returns VaR.

We apply the extreme value approach to the negative daily log returns of AAPL day-of-the-week series from July 3, 1995 to July 2, 2015. Table 6 and Table 7 summarize some estimation results of the shape parameter $\xi$ and VaR. We applyed the maximum likelihood method to estimate parameters of the generalized Pareto distribution for AAPL returns and we calculated the upper 5\% quantile $\mathrm{VaR}$ based on Eq.(12).

Table 6 shows the shape parameter estimates for the day-of-the-week excess returns respectively and corresponding $\mathrm{VaR}$ estimates. In Table 6 , the threshold $\mu$ was chosen via the Hill Plot of the AAPL returns. Around the exceedances $N_{\mu}$ selected by Hill plot, the estimates of the shape parameter are stable for the extremes. Based on the estimated AAPL upper $5 \%$ quantile $\mathrm{VaR}$ results in Table 6 , we found that Friday and Monday $\mathrm{VaR}$ is smaller than that of the rest of the week.

To further investigate systematic weekday differences for AAPL VaR, we also estimate the upper $5 \%$ quantile $\mathrm{VaR}$ via the same threshold $\mu=4.032778$, which is the highest threshold in Table 6. Using the same threshold allows a better comparison of the day-of-the-week VaRs. 
Table 6 Estimated parameters for GPD and VaR of AAPL returns by day-of-the-week.

\begin{tabular}{llllll}
\hline & $\xi$ & $\sigma_{\mu}$ & $\mu$ & $N_{\mu}$ & $\mathrm{VaR}_{0.05}$ \\
\hline Mon. & 0.26884498 & 1.6041546 & 3.795551 & 60 & 4.184124 \\
Tue. & 0.01042914 & 1.76975174 & 4.031559 & 69 & 4.546602 \\
Wed. & 0.3107167 & 1.5597188 & 3.871468 & 69 & 4.317635 \\
Thu. & -0.01453561 & 1.95606434 & 4.032778 & 68 & 4.609665 \\
Fri. & 0.3825541 & 1.4247496 & 3.367018 & 71 & 3.888713 \\
\hline
\end{tabular}

Table 7 Estimated parameters for GPD and VaR of AAPL returns by day-of-the-week via the same threshold $\mu=4.032778$.

\begin{tabular}{lllll}
\hline & $\xi$ & $\sigma_{\mu}$ & $N_{\mu}$ & $\mathrm{VaR}_{0.05}$ \\
\hline Mon. & 0.2725663 & 1.6566470 & 51 & 4.153487 \\
Tue. & 0.01126478 & 1.76707300 & 68 & 4.521224 \\
Wed. & 0.3821768 & 1.4131813 & 64 & 4.347029 \\
Thu. & -0.01453561 & 1.95606434 & 68 & 4.609665 \\
Fri. & 0.4554803 & 1.4688451 & 47 & 3.931803 \\
\hline
\end{tabular}

Table 8 Estimated parameters for GPD and VaR of SPY negative daily log returns by day-of-the-week.

\begin{tabular}{llllll}
\hline & $\xi$ & $\sigma_{\mu}$ & $\mu$ & $N_{\mu}$ & $\mathrm{VaR}_{0.05}$ \\
\hline Mon. & 0.2346827 & 1.0624234 & 1.677897 & 63 & 1.989292 \\
Tue. & 0.1305324 & 0.6817181 & 1.665352 & 68 & 1.856927 \\
Wed. & 0.3743073 & 0.5765108 & 1.780462 & 65 & 1.918262 \\
Thu. & 0.1189783 & 0.8888867 & 1.777264 & 65 & 2.003223 \\
Fri. & -0.06331137 & 0.91646749 & 1.714381 & 64 & 1.93167 \\
\hline
\end{tabular}

The results in Table 7 are mostly consistent with the previous findings in Table 6 . VaRs due to the different tail shapes and the tail fatness of distributions are reflected by the shape parameter $\xi$. The shape parameter measures the speed with which the distribution's tail approaches zero. The fatter the tail, the slower the speed and the higher the shape parameter. From the results in Table 6 and 7, the right tail fatness of Friday excess returns is the highest while that of Thursday is the smallest. The most interesting feature of the results is that the day-of-the-week effect on AAPL VaR is examined. Low Friday and Monday VaR and high Thursday VaR are observed for the AAPL returns.

In capturing the character of AAPL, we present the estimated VaR of SPDR S\&P 500 ETF Trust (SPY) returns in Table 8 , which were obtained via the same approach as applied on AAPL returns. The S\&P 500 Index is composed of five hundred selected stocks in which AAPL weights $3.88 \%$ of total assets. By comparing the VaR of AAPL and SPY, we were able to better capture the characteristic of AAPL.

Table 8 shows that during the test period July 3 , 1995 to July 2, 2015, the upper 5\% quantile VaR of SPY Tuesday and Wednesday returns are smaller than that of the rest of the week. As the top one holding stock of SPY, AAPL naturally has a positive correlation with SPY. However, based on above results, we find the day-of-the-week effect on AAPL $\mathrm{VaR}$ and SPY VaR are different. Moreover, the day-of-the-week VaRs of SPY are much more stable and smaller than that of AAPL. The interesting finding about AAPL is that there is comparatively a high mid-week risk and a low Monday and Friday risk is observed during the test period.

There are many reasons that may cause the day-of-the-week effect on AAPL VaR. Possible explanations for the day-of-the-week effect include the dividends effect, weekend effect and trading activity effect. Apple usually pay its shareholders quarterly dividend on Thursday. It may cause lower trading activity on the following Friday. Due to the 
positive correlation between trading activity and returns, the trading activity during the middle of the week averagely is higher than that of Monday and Friday. In addition, options expiration can influence the overall market as well as specific equities, especially on the last trading day before expiration. AAPL Weeklys option are listed to provide expiration opportunities every week. Weeklys are typically listed on Thursdays and expire on Fridays ${ }^{5}$. Weeklys options can provide opportunities for investors to implement more targeted buying, selling or spreading strategies, which may be the reason why the AAPL returns has an increasing trend during Thursday to Friday. Further research about the exact reasons of weekly effect on AAPL VaR is needed.

\section{Seasonal Effect on AAPL VaR}

Despite finding a weekly pattern in AAPL, one should stress that seasonal effect is by far more relevant in determining stock performance because a three-month period on a financial calendar acts as a basis for the reporting of stock earnings and the paying of dividends. In order to investigate systematic quarterly effects on the stock AAPL, we divide the sample data $\left\{r_{t}\right\}$ into the following four groups:

$$
\left\{r_{t} \mid t \in Q_{i}\right\}, i=1,2,3,4
$$

which are referred to the four quarters AAPL returns. A quarter refers to one-fourth of a year and is typically expressed as Q. Basic tests to examine the seasonal pattern in AAPL returns are carried out next.

Table 9 contains the summary statistics for the four quarter AAPL returns. During the trading period from July 3, 1995 to July 2, 2015, all quarters have positive average return. The first quarter Q1 has the largest average return. Significantly a large range and standard deviations are observed for the Q3 return. The AAPL Q2 return has the smallest standard deviation and average returns than those of the rest

\footnotetext{
${ }^{5}$ Weeklys are not listed if they would expire on a 3rd Friday or if a Quarterly option would expire on the same day
}

seasons. Moreover, the kurtosis indicates the Gaussian behavior of Q2 return since the kurtosis of Q2 return is around 3 .

Table 10 presents that all four quarter AAPL returns are not normally distributed since all the $p$-value of the Shapiro-Wilk test are less than $2.2 e-16$, which are less than the significant level $1 \%$. The correlation test results indicate that quarterly AAPL returns are uncorrelated because the smallest $p$-value among three different lags correlation tests for each group is smaller than the significant level $1 \%$. While for AAPL Q1 returns, the 10 lag and 15 lag Ljung-Box tests $p$ -value indicate the serial correlation of the data set during the test period. However, the 5 lag Ljung-Box test $p$-value is greater than the significant level $1 \%$, which indicate that there is only weak correlation of AAPL Q1 returns. Therefore, we still can process the four datasets as non-normal, independent time series.

Next, we implement the same extreme value approach to VaR on the four seasonal AAPL returns. Table 11 and 12 tell us the same story. Irrespective of our choice to use the different thresholds by Hill plot on the four groups or applying the same threshold, the shape parameter $\xi$ of Q3 returns is the largest which indicates the fattest tail behavior. The upper 5\% quantile VaR of AAPL returns is increasing as the seasons go by in a year. The first season Q1 upper 5\% quantile $\mathrm{VaR}$ is the smallest and the fourth season Q4 upper $5 \%$ quantile $\mathrm{VaR}$ is the largest among four seasonal AAPL returns.

Before giving any explanations of the seasonal effect on AAPL VaR, we take SPY returns as a comparison again to see the characteristic of AAPL.

Table 13 contains estimated upper 5\% quantile VaR of SPY returns by quarter. When comparing results in Table 13 and Table 11, the difference between AAPL VaR and SPY VaR is that the VaR of SPY third quarter returns is the highest while the VaR of AAPL fourth quarter returns is the highest. Moreover, the upper $5 \%$ quantile VaR of seasonal SPY returns is more stable and twice smaller than that of seasonal 
Table 9 Summary statistics of AAPL returns by quarter.

\begin{tabular}{lllllll}
\hline & Mean & Range & Std dev & Skewness & Kurtosis & Obs \\
\hline Q1 & -0.14 & $(-21.36,19.62)$ & 2.98 & -0.25 & 5.7 & 1227 \\
Q2 & -0.04 & $(-12.09,16.30)$ & 2.58 & -0.23 & 3.11 & 1264 \\
Q3 & -0.10 & $(-28.69,73.12)$ & 3.65 & 6.10 & 130.62 & 1272 \\
Q4 & -0.07 & $(-13.37,17.21)$ & 2.87 & -0.08 & 3.33 & 1273 \\
\hline
\end{tabular}

Table 10 Normality and independent test $p$-value of the AAPL returns by quarter

\begin{tabular}{lllll}
\hline & $\mathrm{W}$ & $\mathrm{Q}(5)$ & $\mathrm{Q}(10)$ & $\mathrm{Q}(15)$ \\
\hline Q1 & $<2.2 \mathrm{e}-16$ & 0.01275 & 0.001784 & 0.001235 \\
Q2 & $<2.2 \mathrm{e}-16$ & 0.3114 & 0.2341 & 0.07385 \\
Q3 & $<2.2 \mathrm{e}-16$ & 0.1665 & 0.1343 & 0.01003 \\
Q4 & $<2.2 \mathrm{e}-16$ & 0.6867 & 0.7337 & 0.2699 \\
\hline
\end{tabular}

Table 11 Estimated parameters for GPD and VaR of AAPL returns by quarter.

\begin{tabular}{llllll}
\hline & $\xi$ & $\sigma_{\mu}$ & $\mu$ & $N_{\mu}$ & $\mathrm{VaR}_{0.05}$ \\
\hline Q1 & 0.1582695 & 1.6388243 & 4.110644 & 77 & 3.40719 \\
Q2 & 0.1856602 & 1.0737989 & 3.724132 & 84 & 4.037854 \\
Q3 & 0.4833337 & 1.6498127 & 3.931378 & 79 & 4.308532 \\
Q4 & -0.009111332 & 2.049748142 & 4.038558 & 82 & 4.557201 \\
\hline
\end{tabular}

Table 12 Estimated parameters for GPD and VaR of AAPL returns by quarter via the same threshold 5.

\begin{tabular}{lllll}
\hline & $\xi$ & $\sigma_{\mu}$ & $N_{\mu}$ & $\mathrm{VaR}_{0.05}$ \\
\hline Q1 & 0.1582695 & 1.6388243 & 77 & 3.40719 \\
Q2 & 0.2627277 & 0.9750694 & 63 & 4.107555 \\
Q3 & 0.4524895 & 1.8346705 & 64 & 4.302533 \\
Q4 & -0.04550122 & 2.23903661 & 75 & 4.492442 \\
\hline
\end{tabular}

Table 13 Estimated parameters for GPD and VaR of SPY negative daily log returns from July 3, 1995 to July 2 , 2015 by quarter.

\begin{tabular}{llllll}
\hline & $\xi$ & $\sigma_{\mu}$ & $\mu$ & $N_{\mu}$ & $\mathrm{VaR}_{0.05}$ \\
\hline Q1 & -0.01303372 & 0.81464133 & 1.819322 & 76 & 1.445435 \\
Q2 & 0.1257745 & 0.5497238 & 1.614129 & 78 & 1.693531 \\
Q3 & 0.2794518 & 0.7088729 & 1.956663 & 80 & 2.124614 \\
Q4 & 0.3808598 & 0.8443904 & 1.887993 & 75 & 2.030969 \\
\hline
\end{tabular}

AAPL returns. It is immediately apparent from the above results of test period returns that the seasonal effect on AAPL VaR is different from that on SPY VaR. More importantly, we captured the comparatively high risk in Q4 and low risk in Q1 for AAPL returns during the test period.

Possible explanations for the seasonal effect on AAPL VaR include the tax-motivated trading, economic and political announcements dates concentrated in one part of the season. For instance, Apple often releases its new products, like iPhone, iPad or iMac, during July to November. It may cause large AAPL stock vibration to occur subsequently. Further analysis of these and other explanations is warranted.

Overall, our findings have implications for investors, financial institutions, and futures exchanges. For example, for conservative investors who would prefer lower risk, they can choose to trade during the lower VaR period to avoid potential high loss. The methodology of extreme value approach to VaR can also be used in other stock or asset returns. Finally, it has significant value for investors and regulators in terms of an in depth analysis of the equity market. 


\section{References}

[1] Cross, F. (1973). The behavior of stock prices on Fridays and Mondays. Financial analysts journal, 29(6), 67-69.

[2] Rogalski, R. J. (1984). New findings regarding day?of?the?week returns over trading and non-trading periods: a note. The Journal of Finance, 39(5), 1603-1614.

[3] Baillie, R. T. \& DeGennaro, R. P. (1990). Stock returns and volatility. Journal of financial and Quantitative Analysis, 25(02), 203-214.

[4] Berument, H. \& Kiymaz, H. (2001). The day of the week effect on stock market volatility. Journal of economics and finance, 25(2), 181-193.

[5] Saunders, E. M. (1993). Stock prices and Wall Street weather. American Economic Review, 1337-1345.

[6] Bouman, S. \& Jacobsen, B. (2002). The Halloween indicator, sell in May and go away: Another puzzle. American Economic Review, 1618-1635.

[7] Hirshleifer, D. \& Shumway, T. (2003). Good day sunshine: Stock returns and the weather. Journal of finance, 58(3).

[8] Kamstra, M.J., Kramer, L.A. \& Levi M.D. (2003). Winter blues: Seasonal affective disorder (SAD) and stock market returns. American Economic Review 93, 324-343.

[9] Cao, M. \& Wei, J. (2005). Stock market returns: A note on temperature anomaly. Journal of Banking \& Finance, 29(6), 1559-1573.

[10] Heath, D., Delbaen, F., Eber, J. M., \& Artzner, P. (1999). Coherent Measures of Risk. Mathematical Finance, 9, 203-228.

[11] Jorion, P. (1996). Risk and turnover in the foreign exchange market. The Microstructure of Foreign Exchange Markets (pp. 19-40). University of Chicago Press.

[12] Longin, F. M. (1999). Optimal margin level in futures markets: Extreme price movements. Journal of Futures Markets, 19(2), 127-152.

[13] Longin, F. M. (2000). From value at risk to stress testing: The extreme value approach. Journal of Banking \& Finance, 24(7), 1097-1130.

[14] Tsay, R. S. (2007). Extreme values and their applications in finance.

[15] Riskmetrics: technical document[M]. Morgan Guaranty Trust Company of New York, 1996.

[16] Fisher, R. A., \& Tippett, L. H. C. (1928, April). Limiting forms of the frequency distribution of the largest or smallest member of a sample. In Mathematical Proceedings of the Cambridge Philosophical Society (Vol. 24, No. 02, pp. 180-190). Cambridge University Press.

[17] Gnedenko, B. V. (1943) Sur la distribution limite du terme d'une $S^{\prime}$ é rie alé atoire. Annals of Mathematics, 423-453.

[18] Smith, R. L. (1989). Extreme value analysis of environmental time series: an application to trend detection in ground-level ozone. Statistical Science, 367-377.

[19] Hill, B. M. (1975). A simple general approach to inference about the tail of a distribution. The annals of statistics, 3(5), 1163-1174.

[20] Hosking, J. R. \& Wallis, J. R. (1987). Parameter and quantile estimation for the generalized Pareto distribution. Technometrics, 29(3), 339-349.

[21] Davison, A. C., \& Smith, R. L. (1990). Models for exceedances over high thresholds. Journal of the Royal Statistical Society. Series B (Methodological), 393-442.

[22] Pickands III, J. (1975). Statistical inference using extreme order statistics. the Annals of Statistics, 119-131.

[23] Hendricks, D. (1996). Evaluation of Value-at-Risk Models Using Historical Data (Digest Summary). Economic Policy Review Federal Reserve Bank of New York, 2(1), 39-67.

[24] Kwiatkowski, D., Phillips, P. C., Schmidt, P., \& Shin, Y. (1992). Testing the null hypothesis of stationarity against the alternative of a unit root: How sure are we that economic time series have a unit root?. Journal of econometrics, 54(1), 159-178.

[25] Shapiro, S. S. \& Wilk, M. B. (1965). An analysis of variance test for normality (complete samples). Biometrika, 591-611.

[26] Razali, N. M. \& Wah, Y. B. (2011). Power comparisons of shapiro-wilk, kolmogorov-smirnov, lilliefors and anderson-darling tests. Journal of Statistical Modeling and Analytics, 2(1), 21-33.

[27] Ljung, G. M. \& Box, G. E. (1978). On a measure of lack of fit in time series models. Biometrika, 65(2), 297-303.

[28] Campbell, J. Y. (1993). Understanding risk and return (No. w4554). National Bureau of Economic Research.

[29] Campbell, J. Y. \& Hentschel, L. (1992). No news is good news: An asymmetric model of changing volatility in stock returns. Journal of financial Economics, 31(3), 281-318.

[30] Guo, H. \& Whitelaw, R. F. (2006). Uncovering the risk-return relation in the stock market. The Journal of Finance, 61(3), 1433-1463.

[31] Davison, A. C. \& Smith, R. L. (1990). Models for exceedances over high thresholds. Journal of the Royal Statistical Society. Series B (Methodological), 393-442.

[32] Ameli, A. \& Malekifar, N. (2014). Value at Risk Estimation in Car Insurance by Conditional Heteroskedasticity. Asian Journal of Research in Business Economics and Management, 4(12), 289-297.

[33] Bekaert, G., Erb, C. B., Harvey, C. R., \& Viskanta, T. E. 
(1998). Distributional characteristics of emerging market returns and asset allocation. The Journal of Portfolio Management, 24(2), 102-116.

[34] Kim, T. S., Yoon, J. H., \& Lee, H. K. (2002). Performance of a nonparametric multivariate nearest neighbor model in the prediction of stock index returns. Asia Pacific Management Review, 7(1), 107-118.

[35] Huisman, R., Koedijk, K. G., Kool, C. J. M., \& Palm, F. (2001). Tail-index estimates in small samples. Journal of Business \& Economic Statistics, 19(2), 208-216. 